\title{
An Efficient Framework for Predicting and Recommending M-Commerce Patterns Based on Graph Diffusion Method
}

\author{
R.Priyadharshini \\ SNS College of Technology \\ Coimbatore, India
}

\author{
M.Kalimuthu \\ SNS College of Technology \\ Coimbatore, India
}

\begin{abstract}
Mobile Commerce, also known as M-Commerce or mCommerce, is the ability to conduct commerce using a mobile device. Research is done by Mining and Prediction of Mobile Users' Commerce Behaviors such as their purchase transactions. The problem of PMCP-Mine algorithm has been overcome by the efficient framework based graph diffusion method. The main objective is to construct the graph based diffusion method. Graph is constructed for the items purchased by the Mobile users and then finding the frequently purchased item. By using ranking method, we are ranking the items based on the transactions. Then, by analyzing the mobile users behavior and recommending the ranked items. This framework produces more efficient and accurate item recommendation than the MCE framework.
\end{abstract}

Keywords: Mining, Prediction, Mobile Commerce, diffusion method

\section{INTRODUCTION}

With the rapid advance of wireless communication technology and the increasing popularity of powerful portable devices, mobile users not only can access worldwide information from anywhere at any time but also use their mobile devices to make business transactions easily, e.g., via digital wallet [1]. Meanwhile, the availability of location acquisition technology, e.g., Global Positioning System (GPS), facilitates easy acquisition of a moving trajectory, which records a user movement history. At developing pattern mining and prediction techniques that explore the correlation between the moving behaviors and purchasing transactions of mobile users to explore potential M-Commerce features. Owing to the rapid development of the web 2.0 technology, many stores have made their store information, e.g., business hours, location, and features available online.

Collecting and analysing user trajectories from GPS-enabled devices. When a user enters a building, the user may lose the satellite signal until returning outdoors. By matching user trajectories with store location information, a users' moving sequence among stores in some shop areas can be extracted. The mobile transaction sequence generated by the user is $\{(\mathrm{A},\{\mathrm{i} 1\}), \quad(\mathrm{B}, \varnothing), \quad(\mathrm{C},\{\mathrm{i} 3\}), \quad(\mathrm{D},\{\mathrm{i} 2\})$, $(\mathrm{E}, \varnothing),(\mathrm{F},\{\mathrm{i} 3, \mathrm{i} 4\}),(\mathrm{I}, \varnothing),(\mathrm{K},\{\mathrm{i} 5\})\}$. There is

an entangling relation between moving patterns and purchase patterns since mobile users are moving between stores to shop for desired items.

The moving and purchase patterns of a user can be captured together as mobile commerce patterns for mobile users. To provide this mobile ad hoc advertisement, mining mobile commerce patterns of users and accurately predicts their potential mobile commerce behaviors obviously are essential operations that require more research.

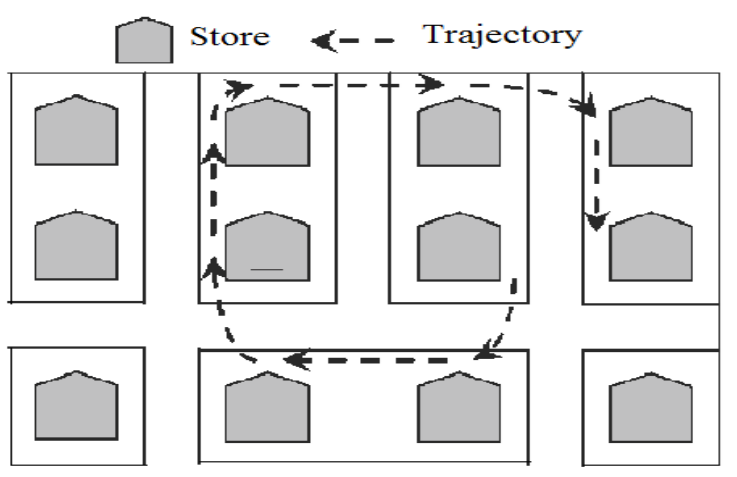

Fig 1 Example of Mobile Transaction Sequence

To capture and obtain a better understanding of mobile users' mobile commerce behaviors, data mining has been widely used for discovering valuable information from complex data sets. They do not reflect the personal behaviors of individual users to support M-Commerce services at a personalized level. Mobile Commerce or M-Commerce, is about the explosion of applications and services that are becoming accessible from Internet-enabled mobile devices. It involves new technologies, services and business models. It is quite different from traditional e-Commerce. Mobile phones impose very different constraints than desktop computers.

\section{PROBLEM DEFINITION}

In the MCE framework, false positive rate is very low and this framework is not more efficient for recommendation. In the graph diffusion framework, the problem is overcome. This framework is more efficient and accurate than the MCE framework.

\section{LITERATURE SURVEY}

Chan Lu, Lee and S. Tseng developed the Mobile Commerce Explorer Framework for mining and prediction of mobile users' movements and purchases [1]. Agrawal and Swami presented an efficient algorithm [2] that generates all significant association rules between items in the database. 
Han, Pei and Yin proposed a novel frequent-pattern tree (FP-tree) structure, which is an extended prefix-tree [3] structure for storing compressed, crucial information about frequent patterns, and develop an efficient FP-tree based mining method, FP-growth, for mining the complete set of frequent patterns by pattern fragment growth.

Hao, Irwin and Michael proposed a novel framework, in which a tree structured graph is constructed for the purchased items by the user. Then based on the log history, finds the suitable item for the mobile users. Then finding the similarity between the log based item and graph based item. If similar the same item will be recommended. If not similar, then the log history based item will be recommended.

\section{EXISTING SYSTEM}

A novel framework for the mobile users' commerce behaviors has been implemented for mining and prediction of mobile users'. MCE framework has been implemented with three components: 1) Similarity Inference Model (SIM) for measuring the similarities among stores and items, 2) Personal Mobile Commerce Pattern Mine (PMCP-Mine) algorithm for efficient discovery of mobile users' Personal Mobile Commerce Patterns (PMCPs), 3) Mobile Commerce Behavior Predictor (MCBP) for prediction of possible mobile user behaviors. In the MCE framework, only frequently moved locations and frequently purchased items are considered. The modules proposed in framework are:

\subsection{Mobile Network Database}

The mobile network database maintains detailed store information which includes locations.

\subsection{Mobile User Data Base}

The Mobile User database maintains detailed mobile user information which include network provider.

\subsection{Applying Data Mining Mechanism}

System has an "offline" mechanism for Similarity inference and PMCPs mining, and an "online" engine for mobile commerce behavior prediction. When mobile users move between the stores, the mobile information which includes user identification, stores, and item purchased are stored in the mobile transaction database. In the offline data mining mechanism, develop the SIM model and the PMCP Mine algorithm to discover the store/item similarities and the PMCPs, respectively. Similarity Inference Model for measuring the similarities among stores and items. Personal Mobile Commerce Pattern-Mine (PMCP-Mine) algorithm is used for efficient discovery of mobile users' Personal Mobile Commerce Patterns.

\subsection{Behavior prediction engine}

In the online prediction engine, implemented a MCBP (Mobile Commerce Behavior Predictor) based on the store and item similarities as well as the mined PMCPs. When a mobile user moves and purchases items among the stores, the next steps will be predicted according to the mobile user's identification and recent mobile transactions. The framework is to support the prediction of next movement and transaction. Mobile Commerce Behavior Predictor for prediction of possible mobile user behaviors.

\subsection{Similarity Inference Model}

A parameter-less data mining model, named Similarity Inference Model, to tackle this task of computing store and item similarities. Before computing the SIM, derive two databases, namely, SID and ISD, from the mobile transaction database. An entry SIDpq in database SID represents that a user has purchased item $q$ in store $p$, while an entry ISDxy in database ISD represents that a user has purchased item $x$ in store $y$. Deriving the SIM to capture the similarity score between stores/items. For every pair of stores or items, SIM assigns them a similarity score. In SIM, used two different inference heuristics for the similarity of stores and items because some stores, such as supermarkets, may provide various types of items.

By applying the same similarity inference heuristics to both of stores and items, various types of items may be seen as similar since different supermarkets are seen as similar. Based on our heuristics, if two stores provide many similar items, the stores are likely to be similar; if two items are sold by many dissimilar stores, the stores are unlikely to be similar. Since the store similarity and item similarity are interdependent, computing those values iteratively. For the store similarity, consider that two stores are more similar if their provided items are more similar. Given two stores sp and sq, compute their similarity SIM (sp; sq) by calculating the average similarity of item sets provided by sp and sq. For every item sold in $\mathrm{sp}$ (and, respectively, sq), first find the most similar item sold in sq (and, respectively, sp). Then, the store similarity can be obtained by averaging all similar item pairs. Therefore, SIM (sp; sq) is defined as

$$
\begin{aligned}
& \operatorname{sim}\left(s_{p}, s_{q}\right)= \sum \varphi \epsilon \Gamma_{s p} \operatorname{Max} \operatorname{Sim}\left(\varphi, \Gamma_{s q}\right)+ \\
& \sum \Upsilon \epsilon \Gamma_{s q} \operatorname{Max} \operatorname{Sim}\left(\Upsilon, \Gamma_{s p}\right)
\end{aligned}
$$

$$
\left|\Gamma_{s p}\right|+\left|\Gamma_{s q}\right|
$$

Where $\operatorname{Max} \operatorname{Sim}(\mathrm{e}, \mathrm{E})=\operatorname{Maxe} \epsilon \mathrm{E} \operatorname{sim}(\mathrm{e}, \mathrm{e}$ ') represents the maximal similarity between e and the element in E. Гsp and $\Gamma$ sq are the sets of items sold in sp and sq, respectively. On the other hand, for the item similarity, consider that two items are less similar if the items are sold by many dissimilar stores. Given two items ix and iy, compute the similarity sim(ix,iy) by calculating the average dissimilarity of store sets that provide ix and iy. For every store providing ix (and, respectively, iy), first find similarity by averaging all dissimilar store pairs.

\subsection{Personal Mobile Commerce Pattern-Mine Algorithm}

The PMCP-Mine algorithm is divided into three main phases: 1) Frequent-Transaction Mining: A FrequentTransaction is a pair of store and items indicating frequently made purchasing transactions. In this phase, first discover all Frequent-Transactions for each user. 2) Mobile Transaction Database Transformation: Based on the all FrequentTransactions, the original mobile transaction database can be reduced by deleting infrequent items. The main purpose is to increase the database scan efficiency for pattern support counting. 3) PMCP Mining: This phase is mining all patterns of length $\mathrm{k}$ from patterns of length $\mathrm{k}-1$ in a bottom-up fashion.

\subsection{Mobile Commerce Behavior Predictor}

MCBP measures the similarity score of every PMCP with a user's recent mobile commerce behavior by taking store and item similarities into account. In MCBP, three ideas are considered: 1) the premises of PMCPs with high similarity to the user's recent mobile commerce behavior are considered as prediction knowledge; 2) more recent mobile commerce behaviors potentially have a greater effect on next mobile commerce behavior predictions and 3) PMCPs with higher support provide greater confidence for predicting users' next mobile commerce behavior. Based on the above ideas, propose a weighted scoring function to evaluate the 
scores of PMCPs. For all PMCPs, calculate their pattern score by the weighted scoring function. The consequence of PMCP with the highest score is used to predict the next mobile commerce behavior.

\subsection{Performance Comparison}

Conduct a series of experiments to evaluate the performance of the proposed framework MCE and its three components, i.e., SIM, PMCP-Mine, and MCBP under various system conditions. The experimental results show that the framework MCE achieves a very high precision in mobile commerce behavior predictions. Besides, the prediction technique MCBP in our MCE framework integrates the mined PMCPs and the similarity information from SIM to achieve superior performs in terms of precision, recall, and F-measure. The experimental results show that the proposed framework and three components are highly accurate under various conditions.

\section{PROPOSED SYSTEM}

\subsection{Construction of data source for user transaction and purchase transaction}

A tree structured graph is constructed for the purchased items by the mobile users. Then the tree constructed for the items. A mobile database is a database that can be connected to by a mobile computing device over a mobile network. The client and server have wireless connections. A cache is maintained to hold frequent data and transactions so that they are not lost due to connection failure. A database is a structured way to organize information. This could be a list of contacts, price information or distance travelled. While those same analysts can't tell us exactly which applications will be the most popular, it is clear that a large percentage will require the use of a database of some sort. Many applications such as databases would require the ability to download information from an information repository and operate on this information even when out of range or disconnected. An example of this is a mobile workforce. In this scenario, a user would require access to update information from files in the home directories on a server or customer records from a database. This type of access and work load generated by such users is different from the traditional workloads seen in client-server systems of today. With the advent of mobile databases, now users can load up their smart phones with mobile databases to exchange mission-critical data remotely without worrying about time or distance. Mobile databases let employees enter data on the fly. Information can be synchronized with a server database at a later time.

\subsection{Building the data source into diffused graph \\ Proposed a parameter-less data mining model,} named Similarity Inference Model based on diffusion graph, to tackle this task of grouping store and item similarities. Before grouping, derive two databases, namely, user and purchase transaction, from the mobile transaction database. Derive the diffusion graph through node formation to capture the similarity nodes with calculation measures of score between data's. Based on heuristics, if two stores provide many similar items, they are likely to be similar; if two items are sold by many dissimilar stores, they are unlikely to be similar. Since the store similarity and item similarity are interdependent, compute them iteratively. Given two stores sp and sq, compute their similarity sim (sp; sq) by calculating the average similarity of item sets provided by $\mathrm{sp}$ and sq. For every item sold in sp (and, respectively, sq), first find the most similar item sold in sq (and, respectively, sp). Then, the store similarity can be obtained by averaging all similar item pairs.

\subsection{A Ranking based Multi-Correlation tensor factorization model on mining web graphs}

In this model, measures the similarity score of every transaction with a user's recent mobile commerce behavior by taking store and item similarities into account. Three ideas are considered in this model: 1) the premise of user node with high similarity to the user's recent mobile commerce behavior are considered as prediction knowledge; 2) more recent mobile commerce behaviors potentially have a greater effect on next mobile commerce behavior predictions; 3) ranking based multi-correlation tensor factorization model with higher support provide greater confidence for predicting user's next mobile commerce behavior.

\subsection{User specific topic modelling through novel diffusion method which propagates similarities \\ User-specific Topic Modelling through novel} diffusion method which Propagates similarities between different nodes (user transaction and Purchase transaction) and generates recommendations to map the purchase relevance and user preference into the same user-specific topic space. In addition to the ternary interrelations, also collect multiple intra-relations among users; assume that two items with high affinities should be mapped close to each other in the learnt factor subspaces. In the following, first introduce how to construct the tag affinity graph, and then incorporate them into the tensor factorization framework. To serve the ranking based optimization scheme, build the affinity graph based on the semantic relevance and context relevance. The context relevance of tag is simply encoded by their weighted co-occurrence in the image collection.

\section{CONCLUSION}

A novel framework namely MCE was proposed for mining and prediction of mobile users' movements and transactions in mobile commerce environments. In the MCE framework were designed with three major techniques: 1) SIM for measuring the similarities among stores and items; 2) PMCP-Mine algorithm for efficiently discovering mobile users' PMCPs; and 3) MCBP for predicting possible mobile user behaviors. To best knowledge, it is the first work that facilitates mining and prediction of personal mobile commerce behaviors that may recommend stores and items previously unknown to a user. To evaluate the performance of the proposed framework and three proposed techniques, conducted a series of experiments.

The experimental results show that the framework MCE achieves a very high precision in mobile commerce behavior predictions. Besides, the prediction technique MCBP in MCE framework integrates the mined PMCPs and the similarity information from SIM to achieve superior performs in terms of precision, recall, and F-measure. The experimental results show that the proposed framework and three components are highly accurate under various conditions. To overcome the problems of many numbers of items recommended in the MCE framework. The Similarity recommendation framework is more efficient than MCE framework system. 


\section{FUTURE ENHANCEMENT}

For the future work, we plan to explore more efficient mobile commerce pattern mining algorithm, design more efficient similarity inference models, and develop profound prediction strategies to further enhance the MCE framework. In addition, we plan to apply the MCE framework to other applications, such as object tracking sensor networks and location based services, aiming to achieve high precision in predicting object behaviors.

\section{REFERENCES}

[1] Eric Hsueh-Chan Lu, Wang-Chien Lee, and Vincent S. Tseng, "A Framework for Personal Mobile Commerce Pattern Mining and Prediction" IEEE transactions on knowledge and data engineering year 2012.

[2] R. Agrawal, T. Imielinski, and A. Swami, "Mining Association Rule between Sets of Items in Large Databases," Proc. ACM SIGMOD Conf. Management of Data, pp. 207-216, May 1993.

[3] J. Han, J. Pei, and Y. Yin, "Mining Frequent Patterns without Candidate Generation," Proc. ACM SIGMOD Conf. Management of Data, pp. 1-12, May 2000.

[4] S.C. Lee, J. Paik, J. Ok, I. Song, and U.M. Kim, "Efficient Mining of User Behaviors by Temporal Mobile Access Patterns," Int'l J. Computer Science Security, vol. 7, no. 2, pp. 285-291, Feb. 2007

[5] V.S. Tseng and K.W. Lin, "Efficient Mining and Prediction of User Behavior Patterns in Mobile Web Systems", Information and Software Technology, vol. 48, no. 6, pp. 357-369, June 2006.

[6] X. Yin, J. Han, P.S. Yu, "LinkClus: Efficient Clustering via Heterogeneous Semantic Links," Proc. Int'l Conf. Very Large Data Bases, pp. 427-438, Aug. 2006.

[7] C.H. Yun and M.S. Chen, "Mining Mobile Sequential Patterns in a Mobile Commerce Environment," IEEE Trans. Systems, Man, and Cybernetics, Part C, vol. 37, no. 2, pp. 278-295, Mar. 2007.

[8] Hao Ma, Irwin King, and Michael Rung-Tsong Lyu, "Mining Web Graphs for Recommendation," IEEE Transactions on Knowledge and Data Engineering, vol.24, No. 6, June 2012.

[9] J. Han and Y. Fu, "Discovery of Multiple-Level Association Rules in Large Database," Proc. Int'l Conf. Very Large Data Bases, pp. 420-431, Sept. 1995.

[10] Y. Zheng, L. Zhang, X. Xie, and W.Y. Ma, "Mining Interesting Location and Travel Sequences from GPS Trajectories," Proc. Int'l World Wide Web Conf., pp. 791-800, Apr. 2009.

[11] Y. Tao, C. Faloutsos, D. Papadias, and B. Liu, "Prediction and Indexing of Moving Objects with Unknown Motion Patterns," Proc. ACM SIGMOD Conf. Management of Data, pp. 611-622, June 2004 